\title{
Contrast-enhanced magnetic resonance (MR) T1 mapping with low-dose gadolinium-diethylenetriamine pentaacetic acid (Gd-DTPA) is promising in identifying clear cell renal cell carcinoma histopathological grade and differentiating fat-poor angiomyolipoma
}

\author{
Shuai Wang, Junheng Li, Diru Zhu, Ting Hua, Binghui Zhao \\ Department of Radiology, Shanghai Tenth People's Hospital, Tongji University School of Medicine, Shanghai 200072, China \\ Correspondence to: Ting Hua; Binghui Zhao. Department of Radiology, Shanghai Tenth People's Hospital, Tongji University School of Medicine, \\ Shanghai 200072, China. Email: huating_2008@sina.com; binghuizhao@163.com.
}

Background: This study aimed to identify clear cell renal cell carcinoma (ccRCC) histopathological grade and differentiate it from fat-poor angiomyolipoma (AML). This was achieved through contrastenhanced magnetic resonance (MR) T1 mapping with intravenous low-dose gadolinium-diethylenetriamine pentaacetic acid (Gd-DTPA).

Methods: In total, 56 consecutive patients received MR scanning between January 2016 and December 2018 using the pre- and post- contrast-enhanced T1 mapping sequences with low-dose Gd-DTPA (0.036 $\mathrm{mmol} / \mathrm{kg}$ ). RCCs were pathologically proven in 40 patients after surgery and graded according to the International Society of Urological Pathology (ISUP) classification system. Ten AMLs were pathologically proven by surgery histopathology and six AMLs were diagnosed by magnetic resonance imaging (MRI). Patients were followed up for more than half a year. The mean T1 values of the renal lesion and ipsilateral normal renal parenchyma were measured before and after Gd-DTPA administration (T1p and T1e). The reduction of T1 value (T1d) and the ratio of its reduction (T1d \%) were calculated and compared.

Results: In 40 ccRCCs, higher-grade [International Society of Urologic Pathology (ISUP) grade 3 and 4] and lower-grade (ISUP grade 1 and 2) ccRCCs were noted in 13 and 27 patients, respectively. The mean T1p was $1,514.8 \pm 139.4 \mathrm{~ms}$ and the mean T1d was $907.7 \pm 193.7 \mathrm{~ms}$ in the higher-grade ccRCCs, which were significantly higher than in the lower-grade ccRCCs ( $\mathrm{T} 1 \mathrm{p}=1,251.7 \pm 151.5 \mathrm{~ms}$ and $\mathrm{T} 1 \mathrm{~d}=648.5 \pm 218.2 \mathrm{~ms}$, respectively; $\mathrm{P}<0.001)$. Fat-poor AMLs had higher T1p $(1,677.3 \pm 104.8 \mathrm{~ms})$ and T1e $(865.6 \pm 251.5 \mathrm{~ms})$ as compared to ccRCCs $(\mathrm{P}<0.001)$. Combined T1p + T1d showed the highest area under the curve (AUC) (0.912) in the differentiation of higher-grade ccRCCs from lower-grade ccRCCs $(\mathrm{P}=0.010)$. Combined T1p + T1e had the highest AUC (0.956) in the differentiation between ccRCCs and fat-poor AMLs $(\mathrm{P}=0.010)$. All T1 mapping metrics could discriminate between normal renal parenchyma and renal lesions $(\mathrm{P}<0.001)$. No significant difference was found in the T1p and T1e at different parts of the ipsilateral normal renal parenchyma. Interobserver agreement for quantitative longitudinal relaxation time in the T1 maps was excellent.

Conclusions: Contrast-enhanced T1 mapping with low-dose Gd-DTPA may provide a more reliable and accurate approach in identifying ccRCCs histopathological grade and differentiating ccRCCs from fat-poor AMLs.

Keywords: Magnetic resonance imaging (MRI); radiologic technology; image enhancement; gadoliniumdiethylenetriamine pentaacetic acid (Gd-DTPA); kidney neoplasms; angiomyolipoma (AML) 
Submitted Aug 28, 2019. Accepted for publication Apr 14, 2020.

doi: 10.21037 /qims-19-723

View this article at: http://dx.doi.org/10.21037/qims-19-723

\section{Introduction}

Renal cell carcinomas (RCCs) are the most common urologic malignancies and can be divided into various subtypes, among which clear cell RCC (ccRCC) is the most common (about 70\%) (1). Different grades of ccRCC exhibit distinct biological behaviors and clinical prognoses $(2,3)$. Minimally invasive therapies, such as percutaneous radiofrequency, cryoablation, microwave, and high-intensity focused ultrasound ablation, have been used as alternatives to surgical treatment for RCCs $(4,5)$. Determination of the tumor grade of ccRCCs by magnetic resonance imaging (MRI) before surgery is vital for clinical decision-making in the management of ccRCCs. MRI is a noninvasive technique that may be used to characterize renal tumors using various functional imaging sequences. In particular, T1 mapping may vary with different diseases, as reflected by the resulting images $(6,7)$. T1 mapping is based on a parametric map in which each pixel represents the T1 spinlattice relaxation time. The $\mathrm{T} 1$ relaxation time, which measures the mobility of molecules, is a molecular signature of healthy or diseased tissues. Recent studies on cardiac MRI showed that T1 mapping was efficient in quantifying the diffuse and focal myocardial edema and fibrosis in patients with myocardial infarction or cardiomyopathy (8-11). In the kidney, T1 mapping can differentiate the cortex and medulla in the normal renal parenchyma (12), and the $\mathrm{T} 1$ relaxation time has been shown to be a reliable marker of injury and can potentially identify pathological changes, including edema, inflammation, and fibrosis (13). Due to the evident T1-shortening effect after intravenous injection of gadolinium-diethylenetriamine pentaacetic acid (Gd-DTPA), the alteration in the T1 relaxation time can be measured quantitatively by $\mathrm{T} 1$ mapping. Contrastenhanced T1 mapping has been reported to be more reliable for predicting the progression of chronic kidney and liver diseases and the degree of differentiation of malignant tumors (14-16).

It has been reported that nephrogenic systemic fibrosis (NSF) and acute anuric renal failure are associated with the use of Gd-based contrast agents (CAs) in patients with underlying renal insufficiencies, with nephrotoxic effects occurring at a dosage of $0.27 \mathrm{mmol} / \mathrm{kg}$ or even below $0.2 \mathrm{mmol} / \mathrm{kg}$ body weight (17-21). The association between the repetitive application of Gd-based (linear) CAs and the Gd deposition in the brain and other organs has also triggered awareness of such issues with Gd-based CAs, and there has been a shift toward techniques that allow for a reduced dose of Gd-based CAs (22-24). As a functional magnetic resonance (MR) technique, T1 mapping has attracted attention for its ability to detect renal injuries due to its reduced CA administration requirements, but few studies have reported which contrast-enhanced T1 mapping is more applicable for characterizing renal neoplasms.

The aim of this study was to investigate the feasibility of preand post-contrast-enhanced T1 mapping with low dose GdDTPA, in order to evaluate the ccRCCs histological grade and differentiate ccRCC from poor-fat angiomyolipoma (AML).

\section{Methods}

\section{Study design and population}

This study was approved by the institutional review board of our hospital. All patients were diagnosed with renal lesions by ultrasonography and received further MRI for clinical examinations. Patients were informed about the purpose, benefits, and risks of these MR scanning protocols, and informed consent was obtained from each patient. Patients with preoperatively suspected ccRCCs based on MRI, who underwent surgery and received histological grading according to the International Society of Urological Pathology (ISUP) criteria, were recruited into this study. Patients with ipsilateral healthy kidneys lacking renal malignancies or complex cysts were also included in a separate healthy cohort. The exclusion criteria were as follows: (I) the quality of MR images was poor, (II) patients were allergic to Gd-DTPA, (III) patients were diagnosed with fat-rich AMLs, (IV) the solid components in the tumor were difficult to characterize. The grade 1 and grade 2 ccRCCs were placed into the lower-grade group, and the grade 3 and grade 4 ccRCCs were placed into the highergrade group, due to limited number of patients with grade 1 $(n=3)$ or grade $4(n=1)$ ccRCCs. 
Table 1 Protocols of magnetic resonance sequences

\begin{tabular}{|c|c|c|c|c|c|}
\hline Sequence & $\begin{array}{l}\text { T1-weighted in-phase or } \\
\text { opposed-phase image }\end{array}$ & T2-weighted image & T2-weighted image & $\begin{array}{l}\text { Contrast-enhanced } \\
\text { VIBE }\end{array}$ & T1 mapping \\
\hline Scan plane & Axial & Axial & Coronal & Axial & Axial \\
\hline TR/TE (ms) & 240/2.31, 3.69 & $1,300 / 89$ & $1,300 / 93$ & $3.92 / 1.39$ & $5.66 / 1.92$ \\
\hline Slice thickness (mm) & 4 & 4 & 4 & 3 & 4 \\
\hline Voxel size $\left(\mathrm{mm}^{3}\right)$ & $2.1 \times 1.5 \times 4$ & $2.1 \times 1.5 \times 4$ & $2.1 \times 1.5 \times 4$ & $1.7 \times 1.2 \times 3$ & $1.1 \times 1.1 \times 4$ \\
\hline Flip angle $\left({ }^{\circ}\right)$ & 70 & 160 & 160 & 9 & $2 / 14$ \\
\hline NEX & 1 & 1 & 1 & 1 & 1 \\
\hline
\end{tabular}

TR, repetition time; TE, echo time; FoV, field of view; NEX, number of excitations; VIBE, volumetric interpolated breath-hold examination.

\section{MRI protocols}

All patients with renal-occupying lesions were scanned continuously with the same 3.0T MRI system (Verio, Siemens Medical Solutions, Germany) using a 16-channel phased-array abdominal coil (Siemens, Germany). The renal MR protocols included axial T1-weighted in- and outphase imaging, axial and coronal T2-weighted imaging, fat saturation volumetric interpolated breath-hold examination (VIBE)-T1-weighted imaging, and native T1 mapping. Multiphase contrast-enhanced VIBE-T1-weighted images were acquired at 20,45 , and $180 \mathrm{~s}$ after intravenous administration of Gd-DTPA. The enhanced T1 mapping was acquired at 90-120 s after Gd-DTPA administration. Each T1 mapping sequence scanning was acquired with about $15 \mathrm{~s}$ of breath-holding. A parallel imaging technique ( $\mathrm{R}$ factor of 2 ) was performed using generalized autocalibrating partially parallel acquisitions (GRAPPA). The duration of each MRI was about $20 \mathrm{~min}$. The detailed MR parameters are listed in Table 1 .

The Gd-DTPA (gadopentetate dimeglumine injection, Xudong-Haipu Pharmaceutical Ltd.) was administered at $0.036 \mathrm{mmol} / \mathrm{kg}$ and intravenously injected at $2.0 \mathrm{~mL} / \mathrm{s}$.

\section{Quantitative MR image analysis}

MR images were analyzed in the workstation (Siemens Syngo Leonardo). T1-relaxation times were calculated on a pixel-by-pixel basis within the regions of interest (ROIs) in a color distribution map. Circular 2-dimensional ROIs were manually selected as the section showing enhanced intensity upon Gd-DTPA injection, and encapsulated or necrotized tumor tissues, vessels, and fat tissues were avoided but identified by a lack of decrease in the signal intensity (SI) on opposed-phase images. Two radiologists, each with 5 years of experience in abdominal MRI, delineated ROIs independently and were blind to the pathology. Each ROI was placed in the same area of a lesion in pre- and post-contrast cases. The ROI for measurements of renal parenchyma was placed in different parts (upper, hilum, lower) of the normal ipsilateral renal parenchyma. The reduction in T1 values (T1d) after enhancement, and the ratio of $\mathrm{T} 1$ reduction ( $\mathrm{T} 1 \mathrm{~d} \%)$ was calculated as follows: $\mathrm{T} 1 \mathrm{~d}=\mathrm{T} 1 \mathrm{p}-\mathrm{T} 1 \mathrm{e} ; \mathrm{T} 1 \mathrm{~d} \%=(\mathrm{T} 1 \mathrm{p}-\mathrm{T} 1 \mathrm{e}) / \mathrm{T} 1 \mathrm{p} \times 100 \% . \mathrm{T} 1$ mapping metrics were performed 3 times by two observers, and the mean values were calculated.

\section{Statistical analysis}

SPSS version 20.0 software (SPSS Inc, Chicago, IL, USA) was used for all statistical analyses. All metrics are presented as the mean \pm standard deviation (SD). Normality tests and homogeneity of variance tests were performed. Oneway analysis of variance (ANOVA) was used to compare the $\mathrm{T} 1$ values at different parts of the normal ipsilateral renal parenchyma. The differences in T1 mapping parameters between ccRCCs and fat-poor AMLs and between lowergrade ccRCCs and higher-grade ccRCCs were compared using the independent samples $t$-test or Mann-Whitney 
Table 2 T1p, T1e, T1d, and T1d\% for renal lesions and normal renal parenchyma

\begin{tabular}{lcccc}
\hline $\begin{array}{l}\text { Renal lesions and } \\
\text { normal parenchyma }\end{array}$ & T1p $(\mathrm{ms})$ & $\mathrm{T} 1 \mathrm{e}(\mathrm{ms})$ & $\mathrm{T} 1 \mathrm{~d}(\mathrm{~ms})$ & $\mathrm{T} 1 \mathrm{~d} \%(\%)$ \\
\hline G1 & $1,062.4 \pm 98.1$ & $649.3 \pm 319.5$ & $413.1 \pm 243.2$ & $40.0 \pm 25.2$ \\
G2 & $1,283.3 \pm 117.9$ & $598.0 \pm 177.3$ & $685.3 \pm 198.00$ & $53.2 \pm 13.5$ \\
G3 & $1,512.2 \pm 151.2$ & $613.1 \pm 171.8$ & $899.1 \pm 208.1$ & $59.3 \pm 10.6$ \\
G4 & $1,546.2$ & 535.4 & $1,010.8$ & 65.3 \\
G1 + G2 & $1,251.7 \pm 151.5$ & $603.2 \pm 190.3$ & $648.5 \pm 218.2$ & $51.7 \pm 14.8$ \\
G3 + G4 & $1,514.8 \pm 139.4$ & $607.1 \pm 159.3$ & $907.7 \pm 193.7$ & $59.8 \pm 9.9$ \\
All ccRCCs & $1,341.9 \pm 182.4$ & $604.8 \pm 180.0$ & $737.1 \pm 241.0$ & $54.1 \pm 14.2$ \\
AML & $1,677.3 \pm 104.8$ & $865.6 \pm 251.5$ & $811.7 \pm 251.4$ & $48.4 \pm 14.3$ \\
Parenchyma & $1,998.3 \pm 129.6$ & $476.8 \pm 124.1$ & $1,521.5 \pm 161.7$ & $76.1 \pm 6.1$ \\
\hline
\end{tabular}

T1p, native T1 values; T1e, enhanced T1 values; T1d, reduction of T1 values; T1d\%, ratio of T1 reduction; ccRCCs, clear cell renal cell carcinomas; AML, angiomyolipoma.

$\mathrm{U}$ test. A receiver operating characteristic (ROC) curve analysis was used to assess the ability of T1 mapping parameters to distinguish fat-poor AMLs from ccRCCs and to distinguish between the different grades of ccRCCs. Interobserver agreement was calculated using the intraclass correlation coefficient (ICC). ICC values of $0.00-0.39$, $0.40-0.59,0.60-0.74$, and $0.75-1.00$ indicated poor, fair, good, and excellent agreement, respectively. A value of $\mathrm{P}$ lower than 0.05 was considered statistically significant.

\section{Results}

\section{Study population and histologic results}

The patients with obvious motion artifacts of MR images or allergy to Gd-DTPA received the CT for clinical examination. Seven fat-rich AMLs were excluded, and ten patients were excluded because the majority of the tumor was encapsulated or the necrotic band had no regions for ROI designation. Finally, 56 patients were included for analysis. There were 40 patients with ccRCC (age: $53.2 \pm 12.4$ years) who underwent partial or radical nephrectomy and subsequent pathological examinations, with 16 patients being diagnosed with poor-fat AML (age: $48.6 \pm 17.4$ years). For AMLs, 10 patients were pathologically diagnosed with fat-poor AMLs after surgery, and 6 fat-poor AML patients were diagnosed and followed up by MRI for more than half a year. The maximum ccRCC diameter ranged from 1.2 to $8.6 \mathrm{~cm}$ (mean: $3.97 \pm 2.15 \mathrm{~cm}$ ). AMLs diameter ranged from 1.5 to $6.7 \mathrm{~cm}$ (mean: $2.89 \pm 1.48 \mathrm{~cm}$ ).
In total, 40 ccRCCs were histologically graded according to the ISUP classification system, including grade 1 in 3 patients, grade 2 in 24 patients, grade 3 in 12 patients, and grade 4 in 1 patient.

\section{T1 mapping with low-dose Gd-DTPA for identifying the solid tissue of renal lesions}

As showed in Table 2, T1 values of renal lesions and normal parenchyma were altered to different extents after administration of low-dose Gd-DTPA $(0.036 \mathrm{mmol} / \mathrm{kg})$. The mean ratio of T1 alteration was $-54.07 \%$ for ccRCCs and $-48.39 \%$ for fat-poor AMLs. There was a significant change between $\mathrm{T} 1 \mathrm{p}$ and $\mathrm{T} 1 \mathrm{e}$ for ccRCCs $(\mathrm{P}<0.001)$ and AMLs $(\mathrm{P}<0.001)$ after Gd-DTPA administration. All T1 mapping metrics could readily discriminate between normal renal parenchyma and renal lesions $(\mathrm{P}<0.001)$. No significant difference was found in the native $\mathrm{T} 1$ and enhanced T1 values at different parts of the ipsilateral normal renal parenchyma $(\mathrm{P}>0.05)$.

\section{Comparison of T1p, T1e, T1d, and T1d\% between fat- poor AMLs and ccRCCs, and between lower-grade CcRCCs and higher-grade ccRCCs}

The T1 mapping parameters are shown in Tables 3,4. The box-and-whisker plots are displayed in Figure 1. Highergrade ccRCCs (ISUP grades 3 and 4) showed significantly higher $\mathrm{T} 1 \mathrm{p}$ and $\mathrm{T} 1 \mathrm{~d}$ values as compared to lower-grade ccRCCs (ISUP grades 1 and 2) $(\mathrm{P}<0.001, \mathrm{P}=0.001)$. The T1e and $\mathrm{T} 1 \mathrm{~d} \%$ between ISUP grades $1-2$ and 3-4 were 
Table 3 Comparative analysis of T1 mapping parameters between fat-poor AMLs and ccRCCs

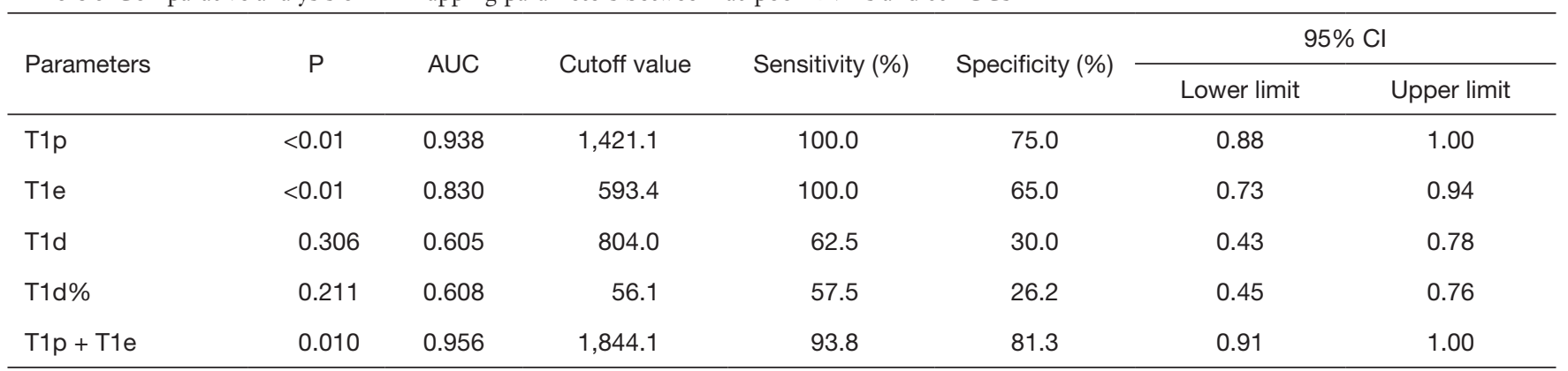

Note: T1p, native T1 values; T1e, enhanced T1 values; T1d, reduction of T1 values; T1d\%, ratio of T1 reduction; T1p + T1e, combined parameter of ROC curve analysis for differentiation between fat-poor AMLs and CCRCCs; ROC, receiver operating characteristic; AUC, area under the curve; $\mathrm{Cl}$, confidence interval; ccRCCs, clear cell renal cell carcinomas; $\mathrm{AML}$, angiomyolipoma.

Table 4 Comparative analysis of T1 mapping parameters between lower-grade ccRCCs and higher-grade ccRCCs

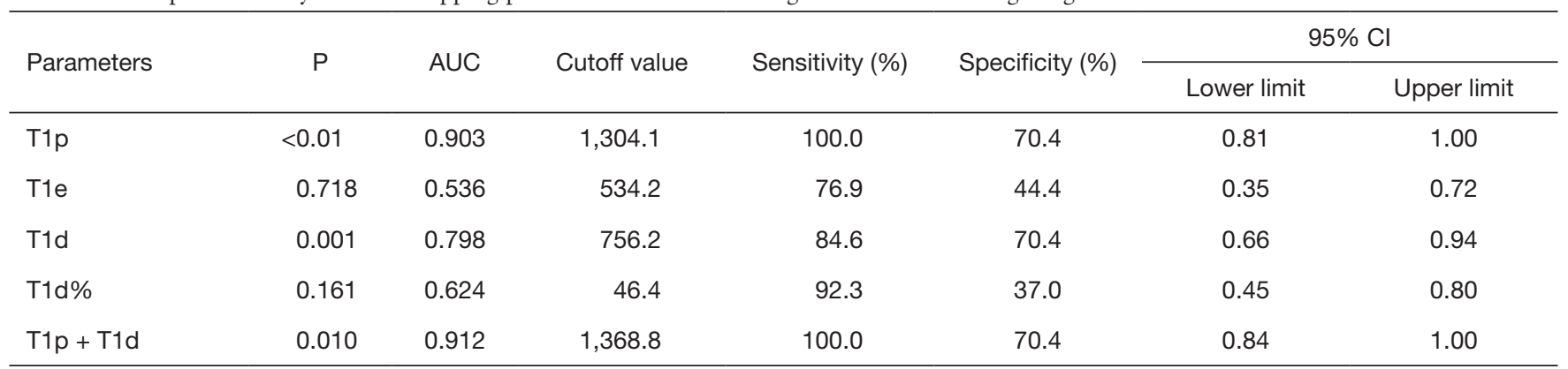

Note: T1p, native T1 values; T1e, enhanced T1 values; T1d, reduction of T1 values; T1d\%, ratio of T1 reduction; T1p + T1d, combined parameter of ROC curve analysis for differentiation between lower-grade ccRCCs and higher-grade ccRCCs; ROC, receiver operating characteristic; ccRCCs, clear cell renal cell carcinomas; AUC, area under the curve; CI, confidence interval.

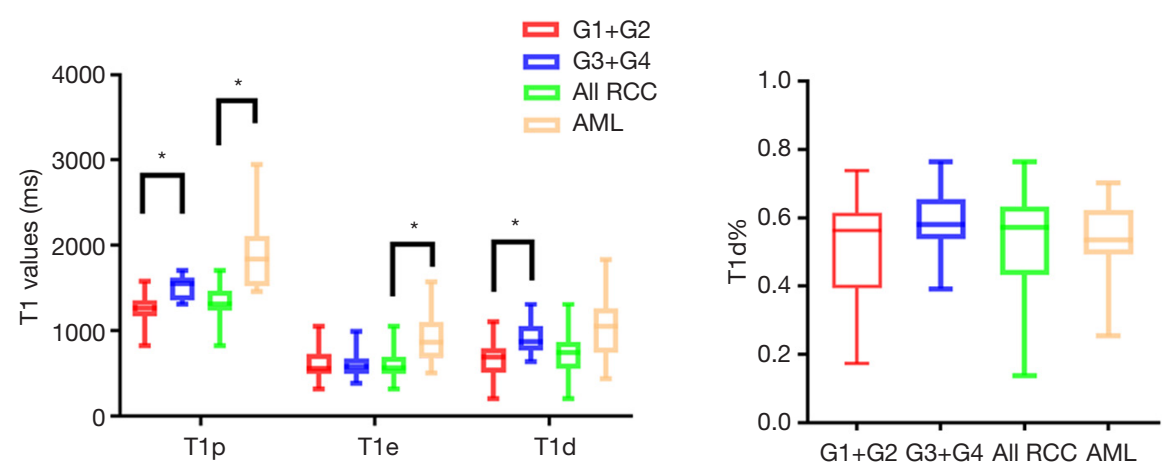

Figure 1 Distribution of T1p, T1e, T1d, and T1d\% across different ISUP grades of ccRCC and AML. *, P<0.05. RCC, renal cell carcinoma; ISUP, International Society of Urological Pathology; ccRCC, clear cell renal cell carcinoma; AML, angiomyolipoma.

similar. Fat-poor AMLs had higher T1p and T1e values as compared to ccRCCs $(\mathrm{P}<0.001)$. The T1d and T1d\% were also comparable between fat-poor AMLs and ccRCCs in the nephrographic phase $(\mathrm{P}>0.05)$.

\section{Diagnostic value of T1 mapping parameters by ROC curve analysis}

ROC curves analysis results are shown in Tables 3,4. The ROC curves (Figure $2 A$ ) of T1p and T1d in discriminating 

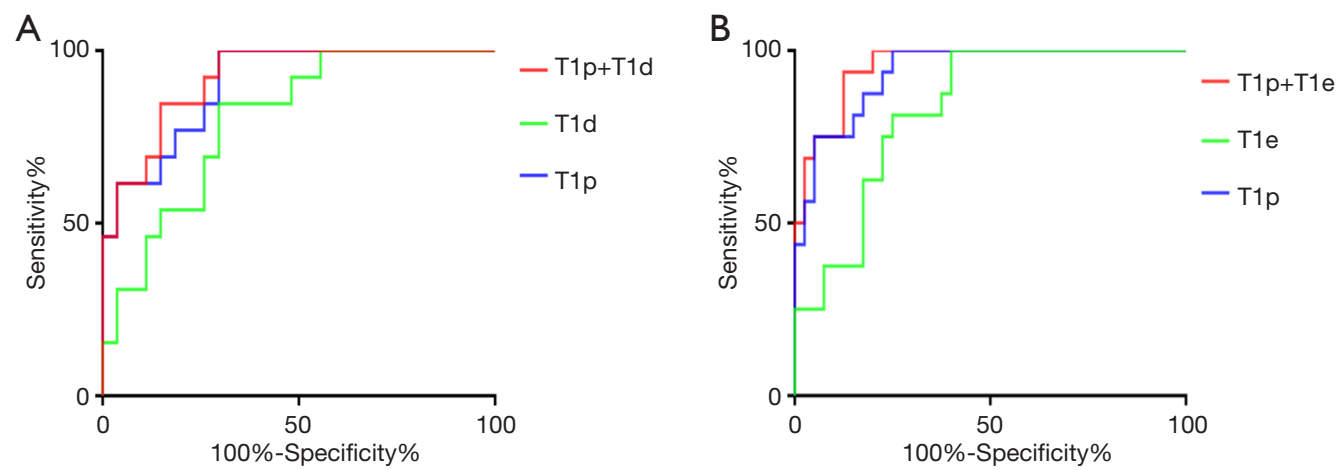

Figure 2 Receiver operating characteristic curves to assess the parameters of T1 mapping for differentiation between fat-poor AMLs and ccRCCs, and between lower-grade ccRCCs and higher-grade ccRCCs. (A) The diagnostic performance of T1 mapping as a binary classifier in discriminating between ISUP grades $1 / 2$ and 3/4; (B) the diagnostic performance of T1 mapping as a binary classifier in discriminating between ccRCC and AML. ISUP, International Society of Urological Pathology; ccRCC, clear cell renal cell carcinoma; AML, angiomyolipoma.

between lower-grade ccRCCs and higher-grade ccRCCs were plotted, and the AUC was 0.903 for T1p and 0.798 for T1d. The best cutoff values for finding higher-grade ccRCCs were 1,304.1 ms for T1p and $756.2 \mathrm{~ms}$ for T1d. For $\mathrm{T} 1 \mathrm{p}$, the sensitivity reached $100.0 \%$, and specificity was $70.4 \%$ in the diagnosis of ccRCCs. For T1d, the sensitivity reached $84.6 \%$, and specificity was $70.4 \%$ in the diagnosis of ccRCCs. Combined T1p + T1d showed the highest AUC (0.912) in differentiating between higher-grade ccRCCs and lower-grade ccRCCs.

The ROC curves of $\mathrm{T} 1 \mathrm{p}$ and $\mathrm{T} 1 \mathrm{e}$ in discriminating between fat-poor AMLs and ccRCCs are shown in Figure $2 B$, and the AUCs were 0.938 for T1p and 0.830 for T1e. The best cutoff values were $1,421.1 \mathrm{~ms}$ for $\mathrm{T} 1 \mathrm{p}$ and $593.4 \mathrm{~ms}$ for T1e. For T1p differentiation between fat-poor AMLs and ccRCC, the sensitivity reached $100.0 \%$, and the specificity was $75.0 \%$, while for T1e, the sensitivity reached $100.0 \%$ and the specificity was $65.0 \%$. The combined $\mathrm{T} 1 \mathrm{p}+\mathrm{T} 1 \mathrm{e}$ had the highest AUC (0.956) in differentiating between ccRCCs and fat-poor AMLs.

\section{Interobserver agreement for quantitative T1 measurements}

In this study, the ICCs of T1 mapping were 0.85 (95\% CI: $0.74-0.91$ ) for T1p and 0.84 (95\% CI: 0.73-0.91) for T1e. These results indicated an excellent interobserver agreement.

\section{Discussion}

This study showed that enhanced T1 mapping with lowdose Gd-DTPA can be used to quantitatively evaluate the renal masses. T1 values of solid renal lesions and normal parenchyma were altered to differing extents after administration of low-dose Gd-DTPA $(0.036 \mathrm{mmol} / \mathrm{kg})$. The mean ratio of $\mathrm{T} 1$ alteration was $-54.07 \%$ for ccRCCs and $-48.39 \%$ for fat-poor AMLs, and there were significant differences between T1p and T1e in ccRCCs and AMLs $(\mathrm{P}<0.001)$ after Gd-DTPA administration. All T1 mapping metrics could readily discriminate between normal renal parenchyma and renal lesions. This study also showed that higher-grade ccRCCs could be distinguished from lower grade ccRCCs based on T1p and T1d values, while combined T1p + T1d had the highest AUC (0.912) for differentiation between higher-grade ccRCCs and lowergrade ccRCCs. Even poor-fat AMLs, which are usually misdiagnosed as RCCs, had higher T1p and T1e values as compared to ccRCCs, while enhanced T1 mapping could provide quantitative parameters to differentiate fatpoor AMLs from ccRCCs. Overall, enhanced T1 mapping has potential as an effective and sensitive technique for identifying ccRCC histological grade and differentiating ccRCCs from fat-poor AMLs.

In the present study, low-dose $(0.036 \mathrm{mmol} / \mathrm{kg}$ weight $)$ Gd-DTPA was intravenously injected for enhanced T1 mapping. The dose was nearly one-third of the standard dose of Gd-DTPA $(0.1 \mathrm{mmol} / \mathrm{kg})$ for contrast-enhanced 
MRI or MR angiography, but it also yielded a significant difference in the T1 value among different renal-occupying lesions. This effect made it easy to identify the solid tissues of renal neoplasms and made the normal renal parenchyma differentiable due to the difference in the blood supply (Figure 3). In addition, patients with renal cancer are more likely to have chronic kidney disease (CKD) at the time of diagnosis and treatment (either radical nephrectomy or partial nephrectomy) than the general population (25). Therefore, clinicians should be aware of the patient's renal function when they plan for surgeries. Low-dose CAs can also significantly reduce the impact of imaging on renal function and decrease the economic burden to patients.

As shown in some studies, MR T1 mapping can provide quantifiable information on longitudinal relaxation via $T 1$ relaxation data and has been applied in several clinical studies (26-28). However, most of the current renal MR sequences only provide SI measurements for semiquantitative or qualitative analysis, which may be affected by various technical factors. The main advantage of T1 mapping is that the T1 longitudinal relaxation time as an absolute value is more stable (29), and it is an intrinsic and fundamental property of a given tissue, reflecting the extracellular expansion and underlying pathophysiological processes and enabling direct T1 quantification (30). Our study showed that enhanced T1 mapping combined with T1 mapping was better than T1 mapping alone and can thus provide quantitative parameters for differentiating ccRCCs from AMLs, increasing the diagnostic efficiency.

ccRCC, the most common subtype of RCC, often shows aggressive behaviors with a relatively higher incidence of metastasis and reduced survival rate as compared to other renal tumors (2,31). Evaluation of tumor grade is critical because higher-grade ccRCCs are often associated with increased aggressiveness, survival, and metastatic potential (32). Accurate assessments of the masses, including the identification of histological subtype and grade, are therefore vital for the appropriate treatment. The technique used in our study is easy to apply in clinical practice as T1 mapping can be done automatically during MRI. In terms of histology, the components of ccRCCs mainly include cells with clear cytoplasm and necrosis, hemorrhage, and cystic degeneration (33). There are two potential reasons why higher-grade ccRCCs show significantly higher T1p values. First, lower-grade ccRCCs are composed of relatively large tumor cells with clear cytoplasm, which means they have more intracellular lipids yielding lower T1p values. Secondly, higher-grade ccRCCs have higher degrees of necrosis, which both occur in the form of macroscopic zones and as micronecrosis. Macroscopic zones are difficult to exclude when delineating ROIs and are therefore included for the ROI analysis, possibly affecting the native $\mathrm{T} 1$ values $(34,35)$. ccRCCs are highly vascularized tumors and can secrete growth factors that act on endothelial cells to stimulate angiogenesis, which helps the tumor recruit blood vessels from adjacent tissues (36). A previous study indicated that higher-grade ccRCCs have higher angiogenic activity than lower-grade ccRCCs (37), while it was also found that microvessel density was reduced with the increase of nuclear grade (36). However, the decreased vascular density can be explained as either a focus of fibrosis or the development of large vessels, which is associated with a poor prognosis (38). Based on the above findings, T1d may increase with the increase of ISUP grade. Therefore, the T1d value may directly reflect the blood supply of tumors and can be used instead of SI to evaluate the degree of enhancement in tissues. Recent studies $(39,40)$ have shown that enhanced T1 mapping may represent an in vivo biomarker for the differentiation of lower- and higher-grade ccRCCs. Also, the native T1 values and MRderived extracellular volumes were significantly associated with the histological collagen volume. In our study, the native $T 1$ values and the reduction of $T 1$ values were determined in different grades of ccRCCs. However, our results showed higher native $\mathrm{T} 1$ values as compared to those previously reported because a higher magnetic field strength was used in this study. These differences may also be explained by the fact that the accuracy, precision, and reproducibility might have been different among different $\mathrm{T} 1$ mapping sequences, possibly affecting $\mathrm{T} 1$ values (41).

AMLs are the most common benign solid renal tumor $(42,43)$. Most AMLs contain some fat tissues that can be recognized on computed tomography (CT) and MR images, and thus these tumors can be easily differentiated from RCCs without biopsies or surgery. However, AMLs with a small amount of fat may or may not show SI on opposedphase MR images, therefore making fat-poor AMLs and fat-invisible AMLs unable to be differentiated from RCCs (44). In particular, fat-poor AMLs cannot be differentiated from ccRCCs rich in cytoplasmic lipids (45-47). Our study indicated that there were significant differences in the T1p and T1e between ccRCCs and fatpoor AMLs $(\mathrm{P}<0.001)$. Fat-poor AMLs had higher T1p values as compared to ccRCCs and had $\mathrm{T} 1 \mathrm{p}$ values similar to adjacent skeletal muscle. In this study, the ROIs were manually delineated as the regions with obviously enhanced 

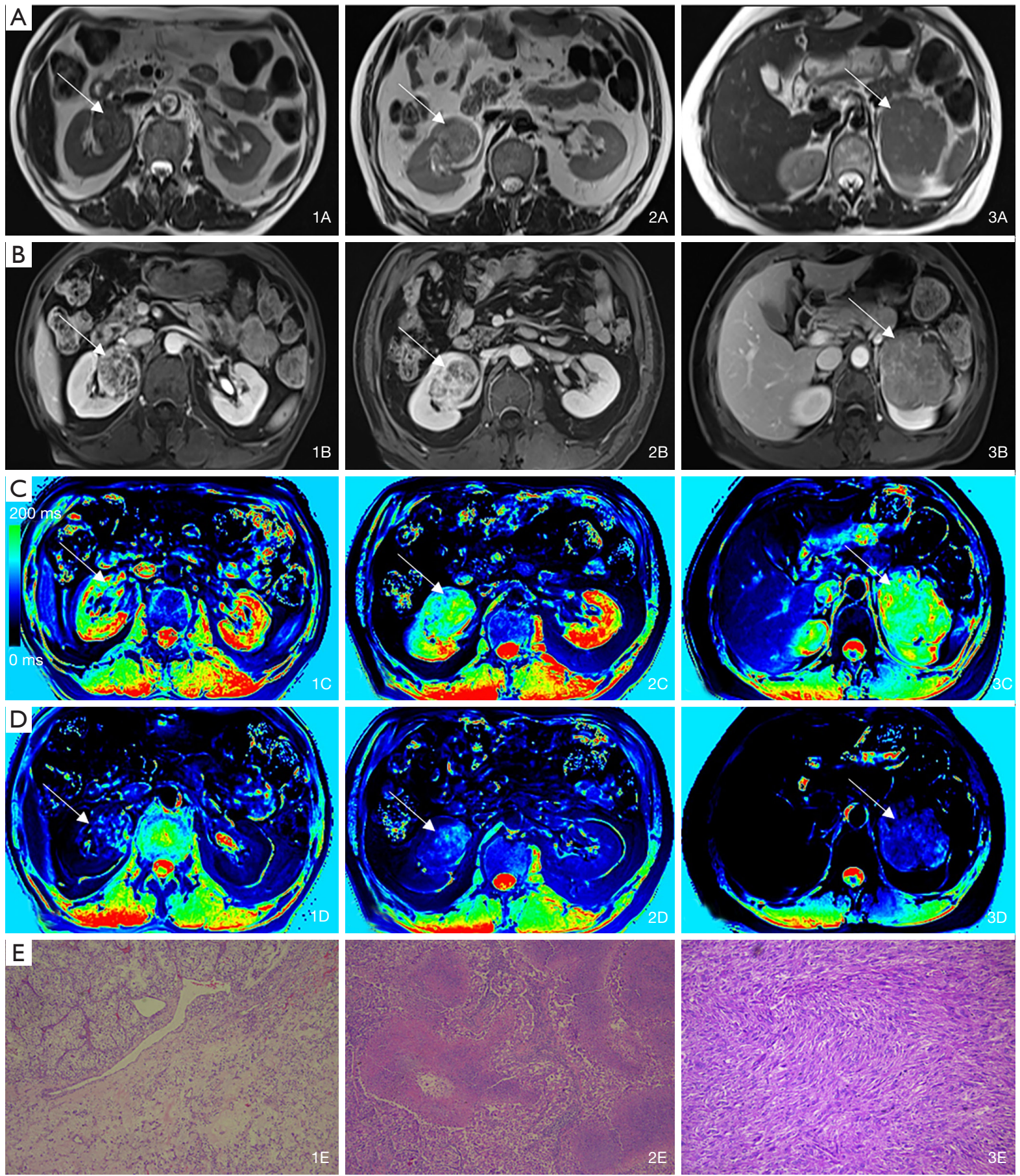

Figure $3 \mathrm{MR}$ and pathological images of different renal lesions. (A) T2 weighted images; (B) enhanced VIBE-T1 images; (C) native T1 value maps; (D) enhanced T1 value maps; (E) corresponding pathological images (hematoxylin and eosin stain, 10×5). White arrows in A-D indicate the location of the lesions. 1A-E: ccRCC (ISUP grade 2) of the right kidney; the lesion appeared iso-intense on T2WI, which reflected a lower native T1 value; enhanced VIBE-T1 showing an inhomogeneous enhancement reflected the reduction of T1 value; tumor cells showed abundant cytoplasm and minimal nuclear irregularity. 2A-E: ccRCC (ISUP grade 4) of the right kidney; native T1 image showed high SI, which reflected a higher T1 value; corresponding enhanced T1 image showed a low T1 value; nuclear pleomorphism and micronecrosis increased with the increase of ISUP grade. 3AE: left renal AML; the lesion appeared iso- or slightly hyperintense signals on T2WI, which reflected a higher T1 value; enhanced T1 maps acquired during the nephrographic phases showing an obvious reduction of T1 value; the components of AML mainly include smooth muscle cells. MR, magnetic resonance; VIBE, volumetric interpolated breath-hold examination; ISUP, International Society of Urological Pathology; ccRCC, clear cell renal cell carcinoma; AML, angiomyolipoma. 
signals, while tumor capsules, necrotic regions, fat tissues, and vessels were avoided. These lower signal regions mainly contain AML smooth muscle components.

In contrast to AMLs, ccRCCs contain abundant intracellular lipids and show relatively hyperintense signals on T1WI, reflecting lower T1 values as compared to fatpoor and fat-invisible AMLs. It has been shown that T1 values may reflect tissue properties. Our study showed ccRCCs had lower T1e values as compared to AMLs, because ccRCCs are often tumors rich in blood supply. However, the T1d value of AMLs is similar to that of ccRCCs. Some studies have reported that AMLs have gradually or persistently enhancing signal (48) while ccRCCs exhibit early wash-in and early washout of contrast material, with most of the contrast material washing out when enhanced T1 mapping is acquired during the nephrographic phase. This may increase T1d values of AML, thus minimizing the difference between AMLs and ccRCCs. Further studies are needed to investigate if T1 mapping at different enhanced phases would be of interest, as such studies could have the potential to quantitatively evaluate enhancement modes.

There are several limitations to this study. First, the sample size was small, especially the number of patients with grade 1 or 4 ccRCCs $(n=4)$, and thus the ccRCCs of different pathological grades were not studied. Second, renal malignant masses in this study were mainly clear cell carcinomas, and other malignant tumors were not included. Thirdly, not all patients with fat-poor AML had a pathological examination, and this might have biased our results. Finally, the reproducibility across different scanners, field strengths, and sequences was not further evaluated. In the future, more studies are needed in which the performance of various $\mathrm{T} 1$ mapping techniques at different magnetic field strengths is evaluated, and in which the histopathological grade of all RCC types is comprehensively assessed.

In conclusion, the novel non-invasive approach of T1 mapping using low-dose Gd-DTPA may provide reliable and accurate parameters for the identification of highergrade ccRCCs and for the differentiation of AMLs from ccRCCs.

\section{Acknowledgments}

Funding: This project was supported by the Fund of the Science and Technology Commission of Shanghai Municipality, China (No. 16411969100).

\section{Footnote}

Conflicts of Interest: All authors have completed the ICMJE uniform disclosure form (available at http://dx.doi. org/10.21037/qims-19-723). The authors have no conflicts of interest to declare.

Ethical Statement: This study was approved by the institutional review board of our hospital.

Open Access Statement: This is an Open Access article distributed in accordance with the Creative Commons Attribution-NonCommercial-NoDerivs 4.0 International License (CC BY-NC-ND 4.0), which permits the noncommercial replication and distribution of the article with the strict proviso that no changes or edits are made and the original work is properly cited (including links to both the formal publication through the relevant DOI and the license). See: https://creativecommons.org/licenses/by-nc-nd/4.0/.

\section{References}

1. Siegel RL, Miller KD, Jemal A. Cancer statistics, 2018. CA Cancer J Clin 2018;68:7-30.

2. Cheville JC, Lohse CM, Zincke H, Weaver AL, Blute ML. Comparisons of outcome and prognostic features among histologic subtypes of renal cell carcinoma. Am J Surg Pathol 2003;27:612-24.

3. Wang K, Cheng J, Wang Y, Wu G. Renal cell carcinoma: preoperative evaluate the grade of histological malignancy using volumetric histogram analysis derived from magnetic resonance diffusion kurtosis imaging. Quant Imaging Med Surg 2019;9:671-80.

4. Gill IS, Remer EM, Hasan WA, Strzempkowski B, Spaliviero M, Steinberg AP, Kaouk JH, Desai MM, Novick AC. Renal cryoablation: outcome at 3 years. J Urol 2005;173:1903-7.

5. Stroup SP, Kopp RP, Derweesh IH. Laparoscopic and percutaneous cryotherapy for renal neoplasms. Panminerva Med 2010;52:331-8.

6. Shah B, Anderson SW, Scalera J, Jara H, Soto JA. Quantitative MR imaging: physical principles and sequence design in abdominal imaging. Radiographics 2011;31:867-80.

7. Winfield JM, Payne GS, deSouza NM. Functional MRI and CT biomarkers in oncology. Eur J Nucl Med Mol Imaging 2015;42:562-78.

8. Puntmann VO, Voigt T, Chen Z, Mayr M, Karim R, 
Rhode K, Pastor A, Carr-White G, Razavi R, Schaeffter T, Nagel E. Native T1 mapping in differentiation of normal myocardium from diffuse disease in hypertrophic and dilated cardiomyopathy. JACC Cardiovasc Imaging 2013;6:475-84.

9. Messroghli DR, Walters K, Plein S, Sparrow P, Friedrich MG, Ridgway JP, Sivananthan MU. Myocardial T1 mapping: application to patients with acute and chronic myocardial infarction. Magn Reson Med 2007;58:34-40.

10. Won S, Davies-Venn C, Liu S, Bluemke DA. Noninvasive imaging of myocardial extracellular matrix for assessment of fibrosis. Curr Opin Cardiol 2013;28:282-9.

11. Salerno M, Kramer CM. Advances in parametric mapping with CMR imaging. JACC Cardiovasc Imaging 2013;6:806-22.

12. de Bazelaire CM, Duhamel GD, Rofsky NM, Alsop DC. MR imaging relaxation times of abdominal and pelvic tissues measured in vivo at $3.0 \mathrm{~T}$ : preliminary results. Radiology 2004;230:652-9.

13. Hueper K, Peperhove M, Rong S, Gerstenberg J, Mengel M, Meier M, Gutberlet M, Tewes S, Barrmeyer A, Chen R, Haller H, Wacker F, Hartung D, Gueler F. T1-mapping for assessment of ischemia-induced acute kidney injury and prediction of chronic kidney disease in mice. Eur Radiol 2014;24:2252-60.

14. Peng Z, Jiang M, Cai H, Chan T, Dong Z, Luo Y, Li ZP, Feng ST. Gd-EOB-DTPA-enhanced magnetic resonance imaging combined with T1 mapping predicts the degree of differentiation in hepatocellular carcinoma. BMC Cancer 2016;16:625.

15. Ding Y, Rao SX, Meng T, Chen C, Li R, Zeng MS. Usefulness of T1 mapping on Gd-EOB-DTPA-enhanced MR imaging in assessment of non-alcoholic fatty liver disease. Eur Radiol 2014;24:959-66.

16. Katsube T, Okada M, Kumano S, Hori M, Imaoka I, Ishii K, Kudo M, Kitagaki H, Murakami T. Estimation of liver function using T1 mapping on Gd-EOB-DTPAenhanced magnetic resonance imaging. Invest Radiol 2011;46:277-83.

17. Grobner T, Prischl FC. Gadolinium and nephrogenic systemic fibrosis. Kidney Int 2007;72:260-4.

18. Wagner B, Drel V, Gorin Y. Pathophysiology of gadolinium-associated systemic fibrosis. Am J Physiol Renal Physiol 2016;311:F1-11.

19. Bahrainwala JZ, Leonberg-Yoo AK, Rudnick MR. Use of Radiocontrast Agents in CKD and ESRD. Semin Dial 2017;30:290-304.

20. Sam AD, 2nd, Morasch MD, Collins J, Song G, Chen R,
Pereles FS. Safety of gadolinium contrast angiography in patients with chronic renal insufficiency. J Vasc Surg 2003;38:313-8.

21. Thomsen HS. Gadolinium-based contrast media may be nephrotoxic even at approved doses. Eur Radiol 2004;14:1654-6.

22. Ramalho M, Ramalho J, Burke LM, Semelka RC. Gadolinium Retention and Toxicity-An Update. Adv Chronic Kidney Dis 2017;24:138-46.

23. Beiderwellen K, Kraff O, Laader A, Maderwald S, Orzada S, Ladd ME, Forsting M, Lauenstein TC, Umutlu L. Contrast enhanced renal MR angiography at 7 Tesla: How much gadolinium do we need? Eur J Radiol 2017;86:76-82.

24. McDonald RJ, McDonald JS, Dai D, Schroeder D, Jentoft ME, Murray DL, Kadirvel R, Eckel LJ, Kallmes DF. Comparison of Gadolinium Concentrations within Multiple Rat Organs after Intravenous Administration of Linear versus Macrocyclic Gadolinium Chelates. Radiology 2017;285:536-45.

25. Russo P. End stage and chronic kidney disease: associations with renal cancer. Front Oncol 2012;2:28.

26. Müller A, Jurcoane A, Kebir S, Ditter P, Schrader F, Herrlinger U, Tzaridis T, Madler B, Schild HH, Glas M, Hattingen E. Quantitative T1-mapping detects cloudyenhancing tumor compartments predicting outcome of patients with glioblastoma. Cancer Med 2017;6:89-99.

27. Yoon JH, Lee JM, Paek M, Han JK, Choi BI. Quantitative assessment of hepatic function: modified look-locker inversion recovery (MOLLI) sequence for T1 mapping on Gd-EOB-DTPA-enhanced liver MR imaging. Eur Radiol 2016;26:1775-82.

28. Okur A, Kantarci M, Kizrak Y, Yildiz S, Pirimoglu B, Karaca L, Ogul H, Sevimli S. Quantitative evaluation of ischemic myocardial scar tissue by unenhanced T1 mapping using 3.0 Tesla MR scanner. Diagn Interv Radiol 2014;20:407-13.

29. Horsthuis K, Nederveen AJ, de Feiter MW, Lavini C, Stokkers PC, Stoker J. Mapping of T1-values and Gadolinium-concentrations in MRI as indicator of disease activity in luminal Crohn's disease: a feasibility study. J Magn Reson Imaging 2009;29:488-93.

30. Haaf P, Garg P, Messroghli DR, Broadbent DA, Greenwood JP, Plein S. Cardiac T1 Mapping and Extracellular Volume (ECV) in clinical practice: a comprehensive review. J Cardiovasc Magn Reson 2016;18:89.

31. Mikami S, Oya M, Mizuno R, Kosaka T, Katsube K, Okada Y. Invasion and metastasis of renal cell carcinoma. 
Med Mol Morphol 2014;47:63-7.

32. Novara G, Martignoni G, Artibani W, Ficarra V. Grading systems in renal cell carcinoma. J Urol 2007;177:430-6.

33. Campbell N, Rosenkrantz AB, Pedrosa I. MRI phenotype in renal cancer: is it clinically relevant? Top Magn Reson Imaging 2014;23:95-115.

34. Delahunt B, McKenney JK, Lohse CM, Leibovich BC, Thompson RH, Boorjian SA, Cheville JC. A novel grading system for clear cell renal cell carcinoma incorporating tumor necrosis. Am J Surg Pathol 2013;37:311-22.

35. Klatte T, Said JW, de Martino M, Larochelle J, Shuch B, Rao JY, Thomas GV, Kabbinavar FF, Belldegrun AS, Pantuck AJ. Presence of tumor necrosis is not a significant predictor of survival in clear cell renal cell carcinoma: higher prognostic accuracy of extent based rather than presence/absence classification. J Urol 2009;181:1558-64; discussion 1563-4.

36. Kinouchi T, Mano M, Matsuoka I, Kodama S, Aoki T, Okamoto M, Yamamura H, Usami M, Takahashi K. Immature tumor angiogenesis in high-grade and highstage renal cell carcinoma. Urology 2003;62:765-70.

37. Baldewijns MM, Thijssen VL, Van den Eynden GG, Van Laere SJ, Bluekens AM, Roskams T, van Poppel H, De Bruine AP, Griffioen AW, Vermeulen PB. High-grade clear cell renal cell carcinoma has a higher angiogenic activity than low-grade renal cell carcinoma based on histomorphological quantification and qRT-PCR mRNA expression profile. Br J Cancer 2007;96:1888-95.

38. Rioux-Leclercq N, Epstein JI, Bansard JY, Turlin B, Patard JJ, Manunta A, Chan T, Ramee MP, Lobel B, Moulinoux JP. Clinical significance of cell proliferation, microvessel density, and CD44 adhesion molecule expression in renal cell carcinoma. Hum Pathol 2001;32:1209-15.

39. Adams LC, Ralla B, Jurmeister P, Bressem KK, Fahlenkamp UL, Hamm B, Busch J, Makowski MR. Native T1 Mapping as an In Vivo Biomarker for the Identification of Higher-Grade Renal Cell Carcinoma: Correlation With Histopathological Findings. Invest Radiol 2019;54:118-28.

40. Adams LC, Jurmeister P, Ralla B, Bressem KK, Fahlenkamp UL, Engel G, Siepmann S, Wagner M, Hamm B, Busch J, Makowski MR. Assessment of the extracellular volume fraction for the grading of clear cell renal cell carcinoma: first results and histopathological findings. Eur Radiol 2019;29:5832-43.

41. Roujol S, Weingartner S, Foppa M, Chow K, Kawaji
K, Ngo LH, Kellman P, Manning WJ, Thompson RB, Nezafat R. Accuracy, precision, and reproducibility of four T1 mapping sequences: a head-to-head comparison of MOLLI, ShMOLLI, SASHA, and SAPPHIRE. Radiology 2014;272:683-9.

42. Fujii Y, Komai Y, Saito K, Iimura Y, Yonese J, Kawakami S, Ishikawa Y, Kumagai J, Kihara K, Fukui I. Incidence of benign pathologic lesions at partial nephrectomy for presumed RCC renal masses: Japanese dual-center experience with 176 consecutive patients. Urology 2008;72:598-602.

43. Jinzaki M, Silverman SG, Akita H, Nagashima Y, Mikami S, Oya M. Renal angiomyolipoma: a radiological classification and update on recent developments in diagnosis and management. Abdom Imaging 2014;39:588-604.

44. Song S, Park BK, Park JJ. New radiologic classification of renal angiomyolipomas. Eur J Radiol 2016;85:1835-42.

45. Jeong CJ, Park BK, Park JJ, Kim CK. Unenhanced CT and MRI Parameters That Can Be Used to Reliably Predict Fat-Invisible Angiomyolipoma. AJR Am J Roentgenol 2016;206:340-7.

46. Jhaveri KS, Elmi A, Hosseini-Nik H, Hedgire S, Evans A, Jewett M, Harisinghani M. Predictive Value of ChemicalShift MRI in Distinguishing Clear Cell Renal Cell Carcinoma From Non-Clear Cell Renal Cell Carcinoma and Minimal-Fat Angiomyolipoma. AJR Am J Roentgenol 2015;205:W79-86.

47. Ferré R, Cornelis F, Verkarre V, Eiss D, Correas JM, Grenier N, Helenon O. Double-echo gradient chemical shift MR imaging fails to differentiate minimal fat renal angiomyolipomas from other homogeneous solid renal tumors. Eur J Radiol 2015;84:360-5.

48. Sasiwimonphan K, Takahashi N, Leibovich BC, Carter RE, Atwell TD, Kawashima A. Small $(<4 \mathrm{~cm})$ renal mass: differentiation of angiomyolipoma without visible fat from renal cell carcinoma utilizing MR imaging. Radiology 2012;263:160-8.

Cite this article as: Wang S, Li J, Zhu D, Hua T, Zhao B. Contrast-enhanced magnetic resonance (MR) T1 mapping with low-dose gadolinium-diethylenetriamine pentaacetic acid (Gd-DTPA) is promising in identifying clear cell renal cell carcinoma histopathological grade and differentiating fat-poor angiomyolipoma. Quant Imaging Med Surg 2020;10(5):988998. doi: 10.21037/qims-19-723 